\title{
Towards a three-dimensional model of wave-ice interaction in the marginal ice zone
}

\author{
C. M. LINTON
}

Department of Mathematical Sciences, Loughborough University, Loughborough LE11 2HD, UK

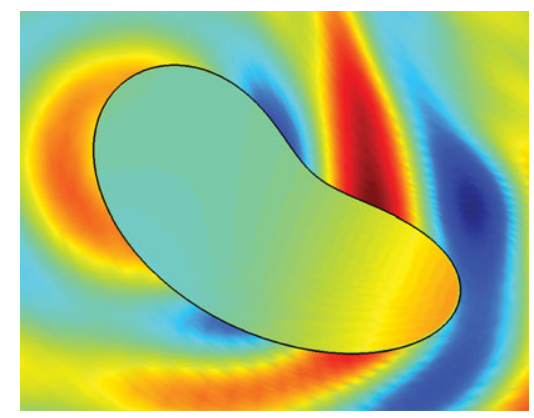

Over the past forty or so years, considerable advances have been made in our understanding of the effects of ocean waves on sea ice, and vice versa, with observations, experiments and theory all playing their part. Recent years have seen the development of ever more sophisticated mathematical models designed to represent the physics more accurately and incorporate new features. What is lacking is an approach to three-dimensional scattering for ice floes that is both accurate and efficient enough to be used as a component in a theory designed to model the passage of directional wave spectra through the marginal ice zone. Bennetts \& Williams (J. Fluid Mech., 2010, this issue, vol. 662, pp. 5-35) have brought together a number of solution techniques honed on simpler problems to provide just such a component.

Key words: sea ice, wave scattering, wave-structure interactions

\section{Introduction}

The interaction between ocean waves and sea ice is a critical factor in the dynamics of the marginal ice zone (MIZ), the intermediate region between the open ocean and an expanse of quasi-continuous sea ice, which typically consists of an assortment of ice floes of varying size and shape. This interaction works both ways. The presence of ice floes on the surface of the ocean affects the wave field through scattering and various dissipative processes and serves to shield the inner sea ice from the impact of the ocean waves. On the other hand the waves incident from the ocean weaken the interior sea ice and cause fractures which then lead to the creation of new floes. Interest in the effect of floating ice on the propagation of water waves dates back to the 19th century and Squire et al. (1995), Squire (2007) provide an extensive review.

The past decade has seen an enormous upsurge in activity in the development of mathematical techniques and models for wave-ice interaction. Partly this is due to the obvious importance of the polar ice caps in relation to the earth's climate, but another driver has been the observation that the same mathematics can be used to describe wave interaction with a class of very large floating structures, (e.g. floating airports) (see Watanabe, Utsunomiya \& Wang 2004). In either case it is usually sufficient to model a thin elastic plate floating on the ocean surface. In their 1995 review, Squire et al. identified a number of areas where knowledge was incomplete and further research desirable. Top of their list was 'developing a fully three-dimensional model for the motion and bending of a solitary ice floe that can be synthesized 
into MIZ-scattering theory to model the passage of directional wave spectra'. Models have certainly become more sophisticated in recent years, with techniques to handle pressure ridges, cracks, leads, variable ice thickness and so on, but this development has largely been in the context of two-dimensional theories.

The situation is made more challenging (and more interesting!) by the fact that it is the diffraction regime (i.e. wavelengths of the same order of magnitude as the floe dimensions) that is the most important. Field measurements (e.g. Robin 1963) show that short waves are rapidly attenuated in the MIZ and for wavelengths greater than about three times the maximum horizontal dimension of a floe, the ice can reasonably be modelled as a rigid structure. Wave-induced ice flexure is greatest in the transitional region and since stresses caused by flexure are the primary cause of floe formation in the MIZ, floes tend to concentrate around this intermediate size.

Some specific three-dimensional geometries have been treated, notably axisymmetric floes (Meylan \& Squire 1996; Bennetts, Biggs \& Porter 2009) and thin cracks (Porter \& Evans 2007) and arbitrary shapes have been handled using shallow-water theory (Sturova 2001), this case being more relevant to the very large floatingstructure problem than the MIZ. Until now the most sophisticated approach to threedimensional scattering by solitary ice floes was that of Meylan (2002), Wang \& Meylan (2004), which incorporates a coupled finite-element/boundary-element method, the former used to compute the vibration modes of the free plate and the latter to solve the water equations. The need to determine the free modes of the floe numerically as part of the solution process is a major drawback if one is interested in combining results from many ice floes to model the MIZ. Moreover, this approach is not suitable for the complementary problem of a hole (known as a polynya) in an otherwise infinite ice sheet.

\section{Overview}

The mathematical boundary-value problem that is typically used to describe oceanwave interaction with a finite, arbitrarily shaped ice floe or polynya is simple to state. It is linear (this is entirely reasonable in most applications though there is no doubt that nonlinear models are required for more extreme seas - work in this area has only just begun; see Hegarty \& Squire 2008), time harmonic, and viscous effects are negligible. A solution is sought for a velocity potential $\varphi$ which satisfies Laplace's equation in the fluid domain, has zero normal derivative on the sea bed (which for simplicity is taken to be horizontal) and then all the difficulties come from the conditions which are applied at the linearized free surface of the water and fluid-ice interface. On those parts of the domain where there is no ice cover we have the standard free surface condition $\partial_{z} \varphi=\sigma \varphi$, where $\sigma$ is related to the frequency of the waves and $z$ is the vertical coordinate, while on the fluid-ice interface we have $\partial_{z} \varphi=\sigma W$ and $\varphi=(1-\sigma d) W+\beta \nabla^{4} W$, in which $W$ is the vertical displacement of the ice, $d$ is the draught of the ice sheet (assumed neutrally buoyant) and $\beta$ accounts for the flexural rigidity of the ice. As well as appropriate radiation conditions, we need conditions which ensure that there is no bending moment or shear stress at the ice edge $\Upsilon$, which is assumed to be a closed smooth curve. These conditions are of the form $\mathscr{B} W=0$ and $\mathscr{S} W=0$ on $\Upsilon$ in which $\mathscr{B}$ and $\mathscr{S}$ are, respectively, second- and third-order differential operators.

The challenge then is to design a procedure for solving this problem which is sufficiently accurate for the results to be useful and efficient enough to be incorporated into a large-scale model of the MIZ. It is too early to say whether or not Bennetts \& 
Williams (2010) have succeeded, largely because such large-scale models are only now being developed (see e.g. Meylan \& Masson 2006; Peter \& Meylan 2009), but there is reason to be optimistic. Over the past decade or so, largely by concentrating on simpler two-dimensional problems, ingenious methods for dealing with the significant technical obstacles that arise in scattering problems of this type have been proposed, tested and refined. Bennetts \& Williams have combined a number of these ideas into a single model which appears to be robust, accurate and efficient.

The first step is to expand the velocity potential in terms of appropriate depth-basis functions, different sets of functions being used in the free-surface and ice-covered regions. The coefficients in these expansions are functions of the horizontal coordinates only and the equations that they must satisfy can be obtained from a variational principle (Porter \& Porter 2004). Crucial to how well this will work in practice is the way the expansions are matched across the ice edge $\Upsilon$, at which there is both a change in depth and upper boundary condition, so as to achieve continuity of pressure and velocity within the fluid. In particular, due to the sharp corner there is a singularity in the velocity field along the lower edge of the ice (i.e. $z=-d, x \in \Upsilon$ ) which, if not accounted for, will lead to an inefficient numerical scheme. Bennetts \& Williams utilize an auxiliary function $u(\boldsymbol{x}, z)$, defined only for $\boldsymbol{x} \in \Upsilon$, which represents the horizontal fluid velocity beneath the ice edge, and expand vertically in terms of weighted Gegenbauer polynomials, in order to capture the aforementioned singularity.

The result of all this is a coupled system of partial differential equations in twohorizontal space dimensions, the unknowns being the coefficients in the various depth expansions that have been made. This is then converted into a system of integral equations posed on $\Upsilon$ using Green's functions which in turn becomes a system of integro-differential equations after the edge conditions $\mathscr{B} W=\mathscr{S} W=0$ are applied. The final step is the discretization of this system which is achieved using a Galerkin technique, the basis functions chosen being sines and cosines. The value in having built in so much of the physics into the solution process is that the truncation parameters required to achieve accurate results are rather small. The authors report that typically of the order of 10 depth modes are required for the velocity potential (in each region), three modes for the horizontal velocity under the ice edge, and between 15 and 30 Fourier modes depending on the incident wavelength. Relative errors of less than $1 \%$ are achieved. In the context of a fully three-dimensional scattering problem this would appear to be an excellent outcome. It should be noted though that the elements of the matrices are given in terms of inner products which need to be computed numerically (except for some special geometries) and that some of these involve quadrature of double integrals.

As in any problem of this type, one needs to be able to reconstruct quantities of physical interest from the computed unknowns. Four are singled out here: diffracted far-field amplitudes (which indicate the directional nature of the scattered response), scattering cross-sections (which provide a measure of the overall strength of the diffracted waves), average curvature of the ice (which relates to the strain and hence to the likelihood of fracture) and maximum wave elevation within polynyas.

\section{Future}

Attempts to accurately model wave-ice interaction in the MIZ are still in the early stage of development. Two-dimensional models have come a long way in the past decade and comparisons with observational data are being made (Kohout \& Meylan 2008), but three-dimensional models which account fully for all significant 
aspects of multiple scattering between ice floes do not exist. Incorporating models for single flows/polynyas such as that described in Bennetts \& Williams (2010) into larger-scale models capable of predicting the effect of large random configurations of such heterogeneities presents a key challenge for the future.

There are many directions in which the Bennetts \& Williams model could be developed. The assumptions of constant ice thickness and constant water depth might be removed as in Bennetts, Biggs \& Porter (2007) and the inclusion of nonsmooth ice edges is another possibility (though a significant theoretical obstacle). Squire (2007) reports that laboratory and field experiments have not kept pace with recent rapid theoretical developments. Validation of the models that are now available would be of enormous value, not least in indicating which, if any, of these possible avenues for further research are actually significant in applications.

\section{References}

Bennetts, L. G., Biggs, N. R. T. \& Porter, D. 2007 A multi-mode approximation to wave scattering by ice sheets of varying thickness. J. Fluid Mech. 579, 413-443.

Bennetts, L. G., Biggs, N. R. T. \& Porter, D. 2009 Wave scattering by an axisymmetric ice floe of varying thickness. IMA J. Appl. Maths 74, 273-295.

Bennetts, L. G. \& Williams, T. D. 2010 Wave scattering by ice floes and polynyas of arbitrary shape. J. Fluid Mech. 662, 5-35.

Hegarty, G. M. \& SQuire, V. A. 2008 A boundary-integral method for the interaction of largeamplitude ocean waves with a compliant floating raft such as a sea-ice floe. J. Engng Maths 62 (4), 355-372.

Kohout, A. L. \& Meylan, M. H. 2008 An elastic plate model for wave attenuation and ice floe breaking in the marginal ice zone. J. Geophys. Res. 113, C09016 (1-17).

Meylan, M. H. 2002 The wave response of ice floes of arbitrary geometry. J. Geophys. Res. 107 (C1), 3005.

Meylan, M. H. \& Masson, D. 2006 A linear Boltzmann equation to model wave scattering in the marginal ice zone. Ocean Model. 11 (3-4), 417-427.

Meylan, M. H. \& SQuire, V. A. 1996 Response of a circular ice floe to ocean waves. J. Geophys. Res. 101 (C4), 8869-8884.

Peter, M. A. \& Meylan, M. H. 2009 Water-wave scattering by vast fields of bodies. SIAM J. Appl. Maths 70 (5), 1567-1586.

Porter, R. \& Evans, D. V. 2007 Diffraction of flexural waves by finite straight cracks in an elastic sheet over water. J. Fluids Struct. 23 (2), 309-327.

Porter, D. \& Porter, R. 2004 Approximations to wave scattering by an ice sheet of variable thickness over undulating bed topography. J. Fluid Mech. 509, 145-179.

Robin, G. De Q. 1963 Wave propagation through fields of pack ice. Phil. Trans. R. Soc. Lond. A 255 (1057), 313-339.

SQuire, V. A. 2007 Of ocean waves and sea-ice revisited. Cold Reg. Sci. Technol. 49 (2), 110-133.

Squire, V. A., Dugan, J. P., Wadhams, P., Rottier, P. J. \& Liu, A. K. 1995 Of ocean waves and sea ice. Annu. Rev. Fluid Mech. 27, 115-168.

Sturova, I. V. 2001 The diffraction of surface waves by an elastic platform floating on shallow water. J. Appl. Maths Mech. 65 (1), 109-117.

Wang, C. D. \& MeYlan, M. H. 2004 A higher-order-coupled boundary element and finite element method for the wave forcing of a floating elastic plate. J. Fluids Struct. 19 (4), 557-572.

Watanabe, E., Utsunomiya, T. \& Wang, C. M. 2004 Hydroelastic analysis of pontoon-type VLFS: a literature survey. Engng Struct. 26, 245-256. 